\title{
Alterations in Circulating Metabolites with Neuroinflammatory Property and Their Impact on Neurological Function in HIV-1-Infected Individuals on Long-Term Suppressive cART
}

Hemalatha Babu, ${ }^{1}$ Gladys Rachel, ${ }^{1,2}$ and Luke Elizabeth Hanna ${ }^{1}$

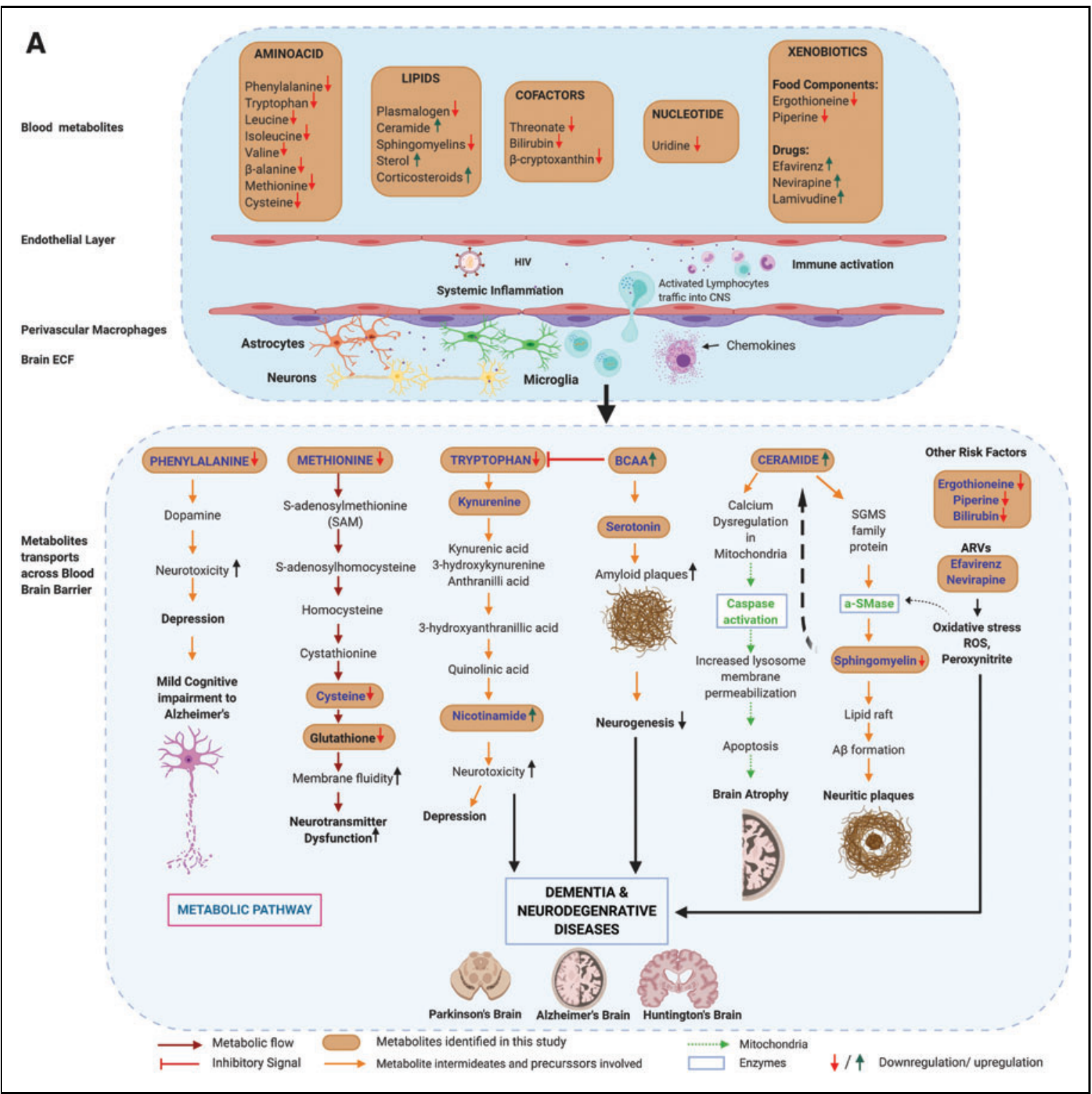

FIG. 1. (A) Metabolic pathways and core signaling cascades implicated in neurodegenerative diseases. (B) Metabolites associated with neuropathology that were found to be significantly altered in HIV-1-infected persons on long-term suppressive cART in this study. (Credit: Created with BioRender.com). cART, combined antiretroviral therapy; BCAA, branched chain aminoacid; a-SMase, acid sphingomyelinase; SGMS, sphingomyelin synthase protein family; ARV, antiretrovirals; ROS, reactive oxygen species; $\mathrm{A} \beta$, amyloid beta plaques; CNS, central nervous system; ECF, extracellular fluid; HIV, human immunodeficiency virus. Color images are available online.

(continued)

\footnotetext{
${ }^{1}$ Department of HIV/AIDS, ICMR - National Institute for Research in Tuberculosis, Chennai, India.

${ }^{2}$ Laboratory Division, ICMR — National Institute of Epidemiology, Chennai, India.
} 


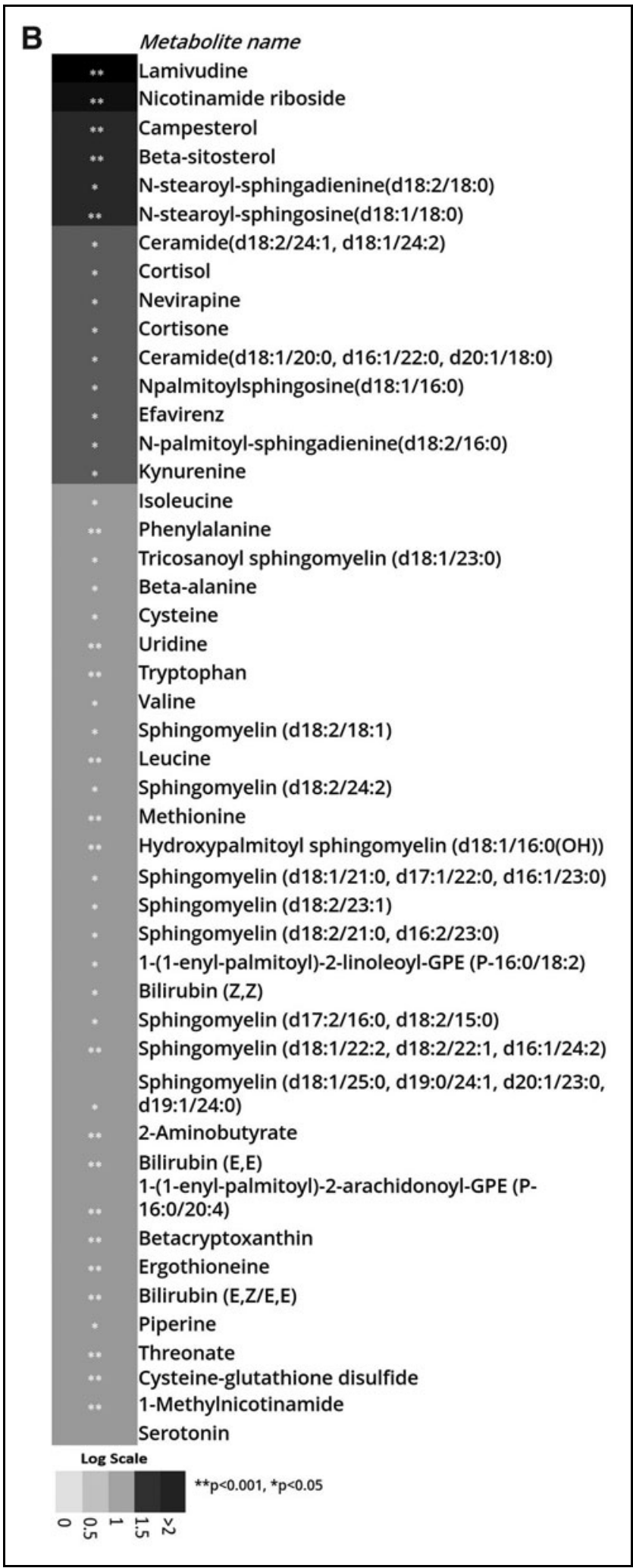

FIG. 1. (Continued.)
$\mathbf{H}^{\mathrm{v}}$ IV-ASSOCIATED NEUROCOGNITIVE DISORDER (HAND) covers a spectrum of neurological and cognitive dysfunction affecting the day-to-day life of persons infected with HIV. Although neurological complications are believed to be significantly reduced upon administration of effective and stable combined antiretroviral therapy (cART), there is evidence to suggest that persons with chronic HIV-1 infection exhibit an increased prevalence of milder forms of cognitive decline despite effective treatment. ${ }^{1}$

In an effort to examine metabolic alterations associated with HIV-1 infection that are known to impact the brain and cause neuropathological conditions, we evaluated a cohort of 22 HIV-1-infected persons with a median age of 45 years on standardized first-line ART regimen (zidovudine/tenofovir and lamivudine with nevirapine/efavirenz) for a median duration of 9 years and compared their metabolic profile with that of an equal number of age-matched HIVuninfected control subjects. Blood samples were collected, processed for plasma separation, and subjected to untargeted metabolomics using ultrahigh-performance liquid chromatography/mass spectrometry/mass spectrometry (UHPLC/ MS/MS). Welch's two-sample $t$-test was used to identify metabolites that were significantly different $(p \leq .05)$ between the two groups.

We observed significant alterations in the levels of several blood metabolites that possess the ability to penetrate the blood-brain barrier and impact neurological pathways in HIV-1-infected persons, clearly indicating a higher risk for neuropathological conditions in these individuals despite effective long-term ART (Fig. 1). Levels of essential amino acids such as phenylalanine, methionine, cysteine, and tryptophan, as well as branched-chain amino acids, known to play an important role in maintaining neurotransmitter and cognitive function, were found to be significantly lower in the HIV-1-positive group. Increased levels of ceramides and decreased levels of sphingomyelin were also observed, indicating an increased risk of mitochondrial dysfunction and $\beta$-amyloid protein formation in the central nervous system. Besides alterations in the classic biomarkers of neurological disease such as tau and amyloid $\beta$ (A $\beta$ ) peptides in cerebrospinal fluid (CSF) and blood, ${ }^{2}$ similar studies have reported alterations in several endogenous and diet-derived metabolites associated with cognitive abnormalities. We also found higher levels of metabolites such as ergothioneine, piperine, and bilirubin, as well as antiretroviral drugs such as nevirapine and efavirenz in HIV-1-infected persons. These metabolites may contribute significantly to neuropathology by increasing oxidative stress and inflammation levels. Elevated systemic inflammation and machine learning predictions of inflammatory and neurological disease risks in HIV-1-infected persons from our previous studies also provide supportive evidence for the observed metabolic abnormalities associated with the central nervous system in HIV-1-infected individuals despite long-term suppressive ART. 3,4

To summarize, we identified alterations in levels of circulating metabolites that could increase the risk of 
developing neurological disorders in HIV-1-infected patients despite viral suppression. Even though neuronal cells are not directly infected by HIV-1, low-level replication in reservoirs such as microglia, accumulation of viral tat protein, blood-brain barrier injury, inflammation, and antiretroviral toxicity along with this metabolic imbalance can cause structural and functional damage to the brain, thus affecting cognitive ability and lead to neurodegeneration in a worst-case scenario.

\section{References}

1. Heaton RK, Clifford DB, Franklin DR Jr, et al.: HIVassociated neurocognitive disorders persist in the era of potent antiretroviral therapy: CHARTER Study. Neurology 2010;75:2087-2096.

2. Frank de Wolf, Mohsen Ghanbari, Silvan Licher, et al.: Plasma tau, neurofilament light chain and amyloid- $\beta$ levels and risk of dementia; a population-based cohort study. Brain 2020;143:1220-1232.
3. Hemalatha Babu, Anoop T. Ambikan, Erin E. Gabriel, et al. Systemic inflammation and the increased risk of inflammaging and age-associated diseases in people living with HIV on long term suppressive antiretroviral therapy. Front Immunol 2019;10:1965.

4. Hemalatha Babu, Maike Sperk, Anoop T, et al.: Plasma metabolic signature and abnormalities in HIV-infected individuals on long-term successful antiretroviral therapy. Metabolites 2019;9:210.

Address correspondence to: Luke Elizabeth Hanna Department of HIV/AIDS ICMR-National Institute for Research in Tuberculosis Chennai 600031

India

E-mail: hanna@nirt.res.in hannatrc@yahoo.com 